\title{
A Frequency Separation Macromodel for System-Level Simulation of RF Circuits
}

\author{
Xin Li, Peng Li, Yang Xu, Robert Dimaggio and Lawrence Pileggi \\ Carnegie Mellon University \\ 5000 Forbes Avenue, Pittsburgh, PA 15213, USA \\ e-mail: \{xinli, pli, yangx, rsd, pileggi\}@ece.cmu.edu
}

\begin{abstract}
In this paper we propose a frequency-separation methodology to generate system-level macromodels for analog and RF circuits. The proposed macromodels are similar in form to those based on Volterra kernel calculations, but are much simpler in terms of characterization and overall model complexity, and can be derived from existing device models. This simplicity is realized by applying some basic assumptions on the form of the input excitations, and via separation of the nonlinearities from the dynamic behavior. In addition, by further separating the ideal model functionality, this macromodel is applicable to strongly nonlinear components such as mixers. While time-varying Volterra series models have been proposed for mixers with a fixed local oscillation (LO) signal, the proposed frequency separation model is completely general and can capture the variations of the LO input during a system-level simulation. The proposed macromodels are demonstrated in a system-level simulation tool based on Simulink for efficient evaluation of the entire RF system and associated components. A GSM receiver system in $0.25 \mu \mathrm{m}$ CMOS process is used to demonstrate the efficacy of these macromodels in our systemlevel simulation environment.
\end{abstract}

\section{Introduction}

Today's remarkable evolution of wireless communication technologies has brought about several new challenges for design of RF circuits. High-performance RF front-ends are required for today's IC communication systems to reduce the signal degradation caused by noise, nonlinear distortion, etc. These design issues have highlighted the need for advanced RF macromodeling techniques for primarily two reasons. First, in top-down designs, high-level simulations based on macromodels can provide fast prediction of system performance, which helps to select proper architectures for circuit implementation and to analyze tradeoffs at the early design stages. Secondly, for bottom-up verification, transistor-level simulation is too expensive in memory space and computation time to facilitate the verification of an entire mixed-signal front-end containing a large number of analog and digital components. Clearly for both cases it is necessary to analyze the interaction of the various components at a higher level of abstraction.

During the past decade, various methods have been developed for RF circuit modeling [1]-[5]. Block diagrams are employed by these approaches as proper macromodel structures because the block diagram form can be easily included in system-level simulation tools such as MATLAB SIMULINK. The modeling approaches proposed in [1]-[5] extract the Volterra kernels directly from the circuit. They are mathematically elegant and have a solid theoretical background based on Volterra series theory [6]-[7]. However, direct Volterra kernel calculations in [1]-[5] require high order derivative information of each nonlinear device, which is not explicitly available in commercial device models, such as the BSIM3 model. Moreover, the modeling approaches proposed in [1]-[5] generate extremely complicated macromodels. For example, [4] converts each nonlinearity in the circuit into several corresponding signal paths, resulting in a great number of coefficient blocks and transfer function blocks in the macromodel. In addition, the modeling techniques in [1]-[5] are only applicable for weakly nonlinear circuits, based on the fundamental limitations of the Volterra series theory in general [2], [6]-[7]. In [8] the authors proposed a timevarying-system-based Volterra series model for the mixer circuit which is a typical example of strongly nonlinear systems. However, the LO input of the mixer circuit must be fixed so that the inputoutput behavior from the RF input to the IF output can be represented by a weakly nonlinear periodically time-varying system. Such a modeling approach, unfortunately, cannot predict the system behaviors when the LO input signal is varying.

In this paper we develop a frequency-separation macromodel for RF circuits that is suitable for system-level simulation. The proposed modeling approach presents several promising features compared with those of other models. First, by considering some basic assumptions on the input excitations, e.g. suppose the circuit input is a single-tone or two-tone signal, we derive a very simple model structure that facilitates efficient system-level evaluations. Note that restricting the input excitation as a single-tone or two-tone signal will not limit the application of our macromodel in most simulation and verification scenarios, since performance specifications of RF circuits are generally measured using a singletone or two-tone test [9]-[10]. Secondly, the model structure proposed in this paper is a frequency-separation one, whereby the nonlinearities are separated from the frequency-dependent (linear dynamic) portions to simplify the modeling problem. As a result, the transfer functions of the linear dynamic blocks can be efficiently extracted and simplified by model order reduction techniques [11][13] with guaranteed stability. Lastly, but most importantly, by further separation of the ideal functionality, our modeling approach can be applied to strongly nonlinear circuits without loss of generality. These models can be then incorporated into a systemlevel simulation environment. Such a modeling capability presently appears to be impractical for Volterra-series-based methodologies.

The remainder of this paper is organized as follows. In Section II we review the background for Volterra series. In Section III we develop our macromodel structure from our frequency-separation methodology, then illustrate the method for model parameter extraction in Section IV. A GSM receiver system in $0.25 \mu \mathrm{m}$ CMOS process is employed in Section $V$ to demonstrate the accuracy and efficiency of the macromodels in system-level simulations.

\section{Background}

A. Volterra Series Theory

Volterra series theory has been introduced in [6]-[7] to analyze and model weakly nonlinear analog systems. A Volterra series describes a nonlinear system by expanding its output response into a number of components at various orders. In general, the Volterra series representation of a nonlinear system can be written as [6]-[7]:

$$
y(t)=\sum_{n=1}^{N} y_{n}(t)
$$

where $y_{n}(t)$ is the nth order response given by

$$
y_{n}(t)=\int_{-\infty}^{+\infty} \cdots \int_{-\infty}^{+\infty} h_{n}\left(\tau_{1}, \cdots, \tau_{n}\right) x\left(t-\tau_{1}\right) \cdots x\left(t-\tau_{n}\right) d \tau_{1} \cdots d \tau_{n}
$$


$x(t)$ and $y(t)$ are system input and output respectively, $h_{n}\left(\tau_{1}, \cdots, \tau_{n}\right)$ is the nth order Volterra kernel, and $N$ is the maximum order of system nonlinearities.

Volterra series theory also provides an explicit formula for the steady-state response of the weakly nonlinear system. Consider the excitation of a multi-tone input defined by

$$
x(t)=\sum_{i=1}^{R}\left|A_{i}\right| \cos \left(\omega_{i} t+\angle A_{i}\right)=\sum_{i=-R, i \neq 0}^{R} \frac{A_{i}}{2} e^{j \omega_{i} t}
$$

where $A_{-i}=A_{i}^{*}$ denotes the conjugate of $A_{i}$, and $\omega_{-i}=-\omega_{i}$, then the $n$th order response of the system can be described as

$$
\begin{aligned}
y_{n}(t)= & \frac{1}{2^{n}} \sum_{i 1=-R, i 1 \neq 0}^{R} \ldots \sum_{i n=-R, i n \neq 0}^{R} A_{i 1} \cdots A_{i n} \\
& \cdot H_{n}\left(\omega_{i 1}, \cdots, \omega_{i n}\right) \cdot e^{j\left(\omega_{i 1}+\cdots+\omega_{i n}\right) t}
\end{aligned}
$$

where

$$
\begin{aligned}
H_{n}\left(\omega_{i 1}, \cdots, \omega_{i n}\right) & =\int_{-\infty}^{+\infty} \cdots \int_{-\infty}^{+\infty} h_{n}\left(\tau_{1}, \cdots, \tau_{n}\right) \\
& \cdot e^{-j\left(\omega_{i 1} \tau_{1}+\cdots+\omega_{i n} \tau_{n}\right)} d \tau_{1} \cdots d \tau_{n}
\end{aligned}
$$

is the nth order kernel transfer function. The steady-state response evaluated by (3)-(5) plays an important role in analyzing and modeling RF circuits, because most performance specifications of RF circuits, such as total harmonic distortion, intermodulation distortion, etc., are defined and measured by their steady-state behaviors.

The Volterra series representation, however, suffers from two limitations. First, it is only applicable for weakly nonlinear systems. For a strongly nonlinear system, the nonlinearity order $N$ in (1) will become very large, or the Volterra series will not even converge in some extreme cases. Secondly, the Volterra series representation for multi-input systems will involve tensor calculations [2], [6], which are quite awkward for many practical applications.

B. Macromodeling by Volterra Series

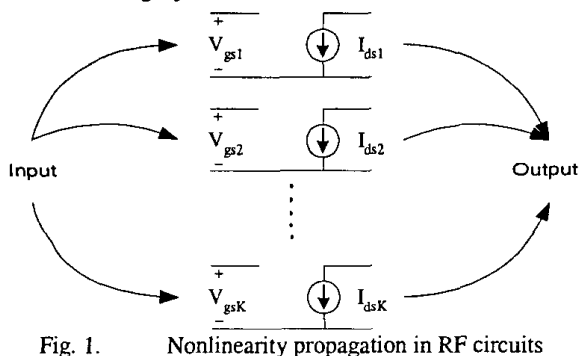

In [4]-[5], a block diagram for Volterra kernels of a nonlinear circuit is proposed for macromodeling. To understand this model structure, consider the 2nd order nonlinearity caused by the nonlinear MOSFETs in a CMOS RF or analog circuit. As shown in Fig. 1, a 2nd order nonlinearity squares the voltage $V_{g s i}$ over its controlling terminal, then multiplies the result with its 2 nd order nonlinearity coefficient to produce a 2 nd order nonlinear current $I_{d s i}$. This current propagates to the output through a linear network, which is simply the linearized circuit at the DC operation point [2], [4]. The total 2nd order output is the sum of the contributions of all those 2 nd order nonlinear currents.

A major disadvantage of such a modeling approach is that every nonlinearity coefficient in the circuit generates several corresponding signal paths in the macromodel. For example, every 2nd order nonlinearity coefficient produces a 2 nd order nonlinear signal path, and two 2nd order nonlinearities can be further combined to generate a 3rd order nonlinear signal path [4] Therefore, the final block diagram model in [4] includes a great number of coefficient blocks and transfer function blocks, even if a simplifying algorithm is applied to eliminate those signal paths with negligible contributions.

\section{Macromodel Structure}

In this section, we first derive a simple frequency-separation macromodel for the LNA. Then, based on that simple model, we further develop a more advanced LNA model, which can predict the 3rd order intermodulation distortion (the only in-band distortion for LNA) accurately. Finally, we extend our modeling method to strongly nonlinear/multi-input circuits and introduce a frequencyseparation macromodel for RF mixers.

\section{A. Simple LNA Model}

As illustrated in the previous section, the modeling approach presented in [4]-[5] produces a complex macromodel which significantly impacts the runtime efficiency of the system-level simulation. Here we propose a much simpler macromodel which considers all signal paths associated with the same nonlinearity order as merged together under single-tone input.

For notational simplicity, we first consider the 2nd order nonlinear effect. If the LNA is excitated by a single-tone input at frequency $\omega$, the 2 nd order harmonic of the nonlinear current $I_{d s i}$ in Fig. 1 is located at frequency $2 \omega$ and its magnitude can be approximately expressed as a function of input amplitude and frequency:

$$
I_{d s i}^{2 n d}=I_{d s i}^{2 n d}(A, s) \quad(i=1,2, \cdots, K)
$$

where $A$ is the input amplitude and $s=j \omega$ specifies the input frequency. According to the Volterra series theory [2], [6]-[7], we simplify (6) as

$$
I_{d s i}^{2 n d}=A^{2} \cdot G_{d s i}(s) \quad(i=1,2, \cdots, K)
$$

because the 2 nd order nonlinear current should be proportional to the square of the input amplitude $A$. Expanding $G_{c s i}(s)$ as a Taylor series

$$
G_{d s i}=G_{d s i 0}+G_{d s i 1} s+G_{d s i 2} s^{2}+\cdots \quad(i=1,2, \cdots, K)
$$

the contribution of the 2 nd order harmonic current $I_{d s i}^{2 n d}$ at the system output can be written as

$$
\begin{aligned}
Y_{d s i} & =A^{2} \cdot\left(G_{d s i 0}+G_{d s i 1} s+\cdots\right) \cdot H_{d s i}(2 s) \\
& =A^{2} \cdot\left(G_{d s i 0}+G_{d s i 1} s+\cdots\right) \cdot \frac{N_{d s i}(2 s)}{D_{d s i}(2 s)} \quad(i=1,2, \cdots, K)
\end{aligned}
$$

Where $H_{d s i}(s)=\frac{N_{d s i}(s)}{D_{d s i}(s)}$ is the linear transfer function from the current source $I_{d s i}$ to the system output. The total 2 nd order harmonic at the system output is the sum of the contributions of all 2nd order harmonic currents, i.e.

$$
Y_{\substack{\text { Second } \\ \text { Order }}}=A^{2} \cdot \sum_{i=1}^{K}\left[\left(G_{d s i 0}+G_{d s i 1} s+\cdots\right) \cdot \frac{N_{d s i}(2 s)}{D_{d s i}(2 s)}\right]
$$

Since different transfer functions of the same linear network share the same poles,

$$
D_{d s 1}(s)=D_{d s 2}(s)=\cdots=D_{d s K}(s)=D_{d s}(s)
$$

Substituting (11) into (10) yields

$$
Y_{\substack{\text { Second } \\ \text { Order }}}=\frac{A^{2} \cdot \sum_{i=1}^{K}\left[\left(G_{d s i 0}+G_{d s i 1} s+\cdots\right) \cdot N_{d s i}(2 s)\right]}{D_{d s}(2 s)}=\frac{A^{2} \cdot N_{d s}(2 s)}{D_{d s}(2 s)}(12)
$$


where $N_{d s}(2 s)$ is a polynomial in $s$ defined by

$$
N_{d s}(2 s)=\sum_{i=1}^{K}\left[\left(G_{d s i 0}+G_{d s i 1} s+\cdots\right) \cdot N_{d s i}(2 s)\right]
$$

The above equations show that all signal paths associated with the 2nd order nonlinearity are merged together. According to (12), we can model the 2 nd order nonlinearity by a square term cascaded with a linear transfer function. In addition, similar results can be obtained for other higher order nonlinearities. For an LNA, we only need to consider the nonlinearities up to 3rd order [4]-[5], because of its weakly nonlinear property, as shown in Fig. 2.

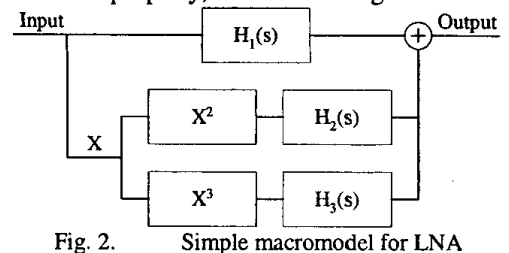

The small-signal transfer function $H_{1}(s)$ in Fig. 2 characterizes the ideal functionality of the LNA, and the other two signal paths are used to predict its 2 nd and 3 rd order nonlinearity respectively. The ideal functionality is separated from the nonideal effects in our macromodel. Furthermore, the nonlinear blocks $X^{2}$ and $X^{3}$ in Fig. 2 are not frequency-dependent, which implies the frequency-separation property of the proposed LNA model.

The simple LNA model in Fig. 2 can be considered as an extension of the Hammerstein model in system identification community [14]. The Hammerstein model in [14] represents the nonlinear dynamic system as a static nonlinear function followed by a linear transfer function, i.e. the same transfer function is used for different order nonlinearities. However, from the analysis in (6)(13), we know the dynamic behaviors of different order nonlinearities are different in general, because the polynomial coefficients in (8) vary for different order nonlinearities. Therefore, in Fig. 2, we employ different transfer functions $\mathrm{H}_{2}(s)$ and $\mathrm{H}_{3}(s)$ to characterize the nonlinearities $X^{2}$ and $X^{3}$ respectively.

The accuracy of this LNA model can be justified by the following theorem.

Theorem 1:

If (a) the LNA circuit is a weakly nonlinear system and its maximum order of nonlinearity is equal to 3 ;

(b) the LNA circuit is a narrow band system and its bandwidth is much smaller than the center frequency;

(c) the LNA circuit is driven by a single-tone sinusoidal input at frequency $\omega$, where $\omega$ is located in the passband of the LNA circuit;

then there exist a set of transfer functions $\mathrm{H}_{1}(s), \mathrm{H}_{2}(\mathrm{~s})$ and $\mathrm{H}_{3}(\mathrm{~s})$ such that the steady-state response generated by the macromodel in Fig. 2 is identical to the steady-state response of the original LNA circuit at the frequencies $\omega, 2 \omega$ and $3 \omega$.

The detailed proof of the theorem can be found in [15]. Note that our macromodel is accurate only for steady-state response and the DC component generated by the macromodel is not guaranteed to be equal to that of the original circuit. However, this is not an important issue, since most performance specifications of RF circuits are measured by their steady-state behaviors and the DC component is generally not included in evaluating the circuit performance [9][10]. For example, the DC component is not used when the total harmonic distortion of the LNA circuit is measured [2].

\section{B. Advanced LNA Model}

The simple LNA model displayed in Fig. 2 is a sufficient one to estimate the circuit response with single-tone input; however, it cannot accurately predict circuit behaviors under multi-tone excitations. This issue can be illustrated by the following example.

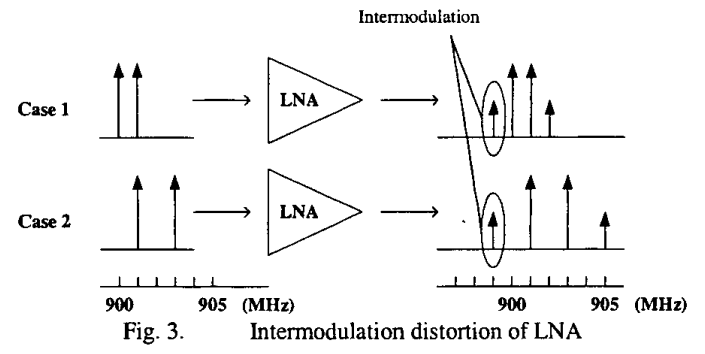

Consider a two-tone input case where the two input frequencies are $900 \mathrm{MHz}$ and $901 \mathrm{MHz}$ respectively and the input amplitudes are both a constant value A. As shown in Fig. 3, the LNA circuit will produce a harmonic component at $899 \mathrm{MHz}$ due to its 3rd order nonlinearity. Now, consider another two-tone input case in which the two input frequencies are $901 \mathrm{MHz}$ and $903 \mathrm{MHz}$ and the input amplitudes are still equal to A. Again, a harmonic component at $899 \mathrm{MHz}$ is generated by the LNA circuit as intermodulation distortion. Based on the macromodel in Fig. 2, the harmonic components at $899 \mathrm{MHz}$ shall be identical in the above two cases, because the linear transfer function $\mathrm{H}_{3}(s)$ in the macromodel presents the same attenuation at the same frequency $899 \mathrm{MHz}$. For the original LNA circuit, however, the intermodulation distortions at $899 \mathrm{MHz}$ shall be different in these two cases. For instance, the RLC network for input impedance match in the LNA circuit will attenuate the input signal differently at different input frequencies [9]-[10], and therefore produce different intermodulation distortions in the above two cases.

This simple example indicates an important fact that the macromodel in Fig. 2 only exhibits different attenuations at different output frequencies. It performs the same operation on input signals at all frequencies, because there doesn't exist any frequencydependent block at the input port of the model. In order to eliminate such a limitation, we add a pre-filter before the nonlinear blocks $X^{2}$ and $X^{3}$, and result in the following advanced LNA model shown in Fig. 4.

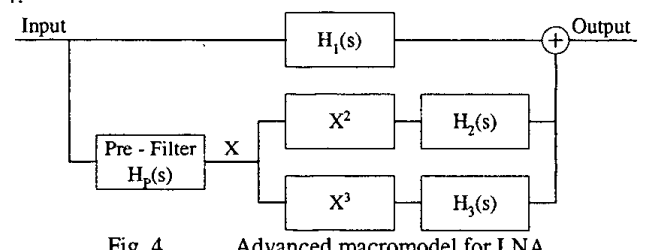

The accuracy of the LNA model in Fig. 4 is justified in a manner similar to that in Theorem 1. Details can be found in [15].

It should be noted that the modified macromodel in Fig. 4 can only estimate the 3 rd order intermodulation distortion accurately under two-tone input. Such a restriction will not limit the application of our macromodel in most practical cases, since the intermodulation distortion of LNA is only simulated and verified using a two-tone input [9]-[10].

\section{Mixer Model}

The differences between the mixer and the LNA lie in the following two points. First, a mixer has two input ports, RF input 
and LO input. This requires extension of the nonlinear blocks in Fig. 4 to two-variable power functions, i.e. $X Y, X^{2} Y, X Y^{2}$, etc. $X$ and $Y$ correspond to the RF input and the LO input respectively. Second, a mixer is a strongly nonlinear system because the LO signal is chosen large enough to drive the transistor switch on and off periodically [9]. The ideal functionality of a mixer, therefore, is equivalent to multiplying the RF signal by a square wave $\operatorname{sign}(\mathrm{LO})$, where $\operatorname{sign}(\bullet)$ denotes the signum function [9]. In such cases, the Volterra series representation converges very slowly and cannot be applied as a model directly. Considering the ideal functionality of the mixer circuit, we add some additional signum function blocks, $X \cdot \operatorname{sign}(Y), X^{2} \cdot \operatorname{sign}(Y)$, etc., into the mixer model. With this modification, the strongly nonlinear property of the mixer circuit can be captured efficiently.

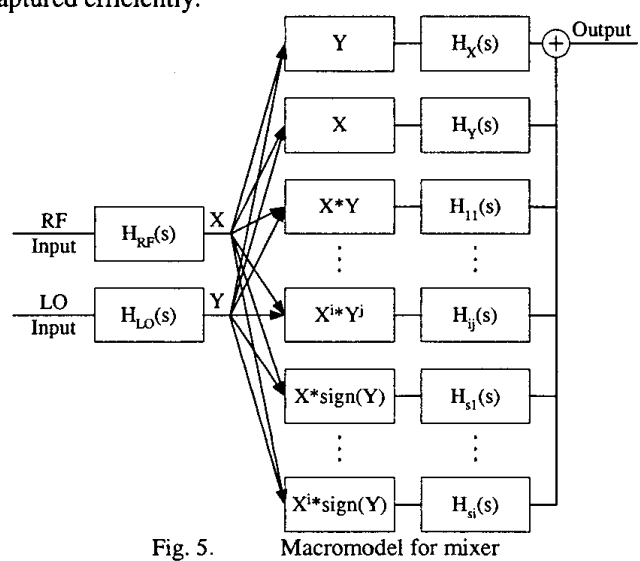

The result is the mixer model shown in Fig. 5. Note that the signal path $X \cdot \operatorname{sign}(Y)$ in the macromodel now represents the ideal functionality and all other paths contribute to the nonideal effects in the mixer circuit. The mixer circuit is regarded as a strongly nonlinear system in general, because the Volterra series representation will apply a great number of high order terms for accurately expanding the signum function $\operatorname{Sign}(Y)$. However, the proposed macromodel in Fig. 5 includes the strongly nonlinear function $\operatorname{Sign}(Y)$ directly. Then, after the strong nonlinearity of the mixer circuit is separated, the remainder input-output relation is weakly nonlinear which is characterized by the power functions $X Y, X^{2} Y, X Y^{2}$, etc in our model. Under such a model representation, the behavior of the macromodel converges to the actual behavior of the original mixer circuit very quickly, as $i$ and $j$ increase. In many applications, it is sufficient to choose the nonlinearity order $i$ and $j$ in Fig. 5 up to 3 .

Finally, it is worth mentioning that we model the mixer circuit as a strongly nonlinear two-input system in this paper, but not as a simple weakly nonlinear single-input time-varying system in [8]. Therefore, the proposed model structure in Fig. 5 is general and can accommodate changing values for the LO input during system-level simulations.

\section{Model Parameter Extraction}

Recall from Section 2.2 that all nonlinear currents $I_{d s i}$ at different nonlinearity orders propagate to the circuit output through the same linear network. In addition, different transfer functions of the same linear network share the same poles, as described in (11). Hence, we use the same poles for all transfer functions in our macromodel. For the purpose of model simplicity and stability, order reduction techniques [11]-[13] are employed to extract and simplify the transfer functions of the linearized circuit at the DC operation point, resulting in a compact group of stable poles.

The zeros in the macromodel can be obtained simply by a datafitting approach because they will not influence the model stability. The least-square approach [14] is employed in this paper to estimate the optimum values of the zeros based on steady-state response data. We first build a set of nonlinear equations using steady-state simulation results at different input amplitudes and frequencies, then nonlinear iteration schemes, such as the Newton-Raphson method, are employed to solve these equations and produce correct zero values.

It is important to note that no high order derivative information is required during our process of model parameter extraction. From this point of view, our macromodeling scheme is not restricted to special device models, as is the case for direct Volterra kernel calculations.

\section{Simulation Results}

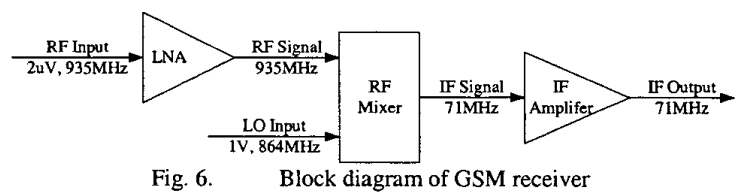

Shown in Fig. 6 is a GSM receiver system in $0.25 \mu \mathrm{m}$ CMOS process which is employed in this section to demonstrate the accuracy and efficiency of the proposed macromodels in systemlevel simulations. For modeling and simulating the entire GSM receiver system we first represent each component in the system, i.e. low noise amplifier, RF mixer and IF amplifier, by a frequencyseparation macromodel. Next, we connect all these components together and construct a signal flow graph in our system-level simulation tool. Such a signal-flow-graph-based GSM receiver is then simulated by our tool to verify its performance at the system level. All the simulations are run on a Pentium IV - $1.4 \mathrm{GHz}$ computer with $256 \mathrm{MB}$ of memory.

A. Low Noise Amplifier

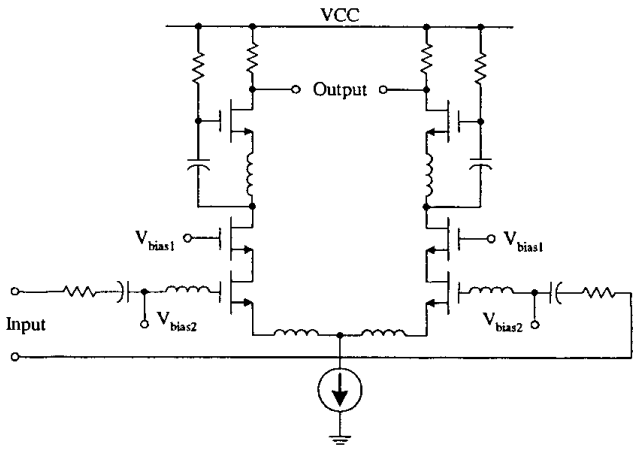

Fig. 7. Circuit schematic of LNA

Fig. 7 shows the circuit schematic of an LNA that includes 6 MOS transistors. This unusually large number of transistors for an LNA is a good example since it produces highly complex nonlinear behaviors to test the proposed macromodel for nonlinear distortions. The center frequency of the LNA is $900 \mathrm{MHz}$ and its small-signal gain is around 16 at the center frequency. Employing the macromodel structure in Fig. 4 we extract the zeros and poles of the transfer functions $H_{P}(s), H_{1}(s), H_{2}(s)$ and $H_{3}(s)$ with order set 
to 11 for this example.

We run steady-state analysis and test the developed macromodel using a single-tone input with an amplitude range of $2 \mu \mathrm{V} \sim 20 \mu \mathrm{V}$ and frequency range of $700 \mathrm{MHz} \sim 1100 \mathrm{MHz}$. The maximum, minimum and average modeling errors are respectively $1.36 \%, 0.53 \%$ and $0.85 \%$. Here, the modeling error is defined by

$$
\text { Error }=\sqrt{\left[\sum_{i=0}^{N}\left|\hat{Y}_{i}-Y_{i}\right|^{2}\right] /\left[\sum_{i=0}^{N}\left|\hat{Y}_{i}\right|^{2}\right]}
$$

where $\hat{Y}_{i}$ is the ith order output harmonic obtained by the original LNA circuit and $Y_{i}$ is the corresponding result estimated by the macromodel. In (14), $N$ is chosen large enough so that those high order harmonics beyond $N$ are negligible.

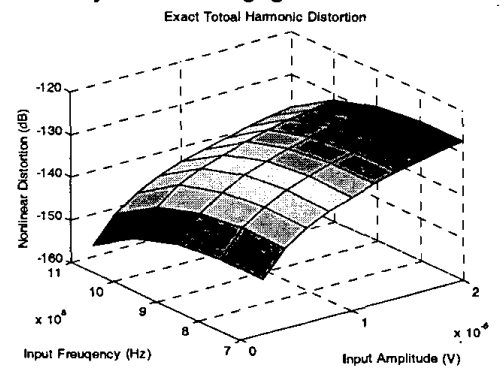

Fig. 8. Exact total harmonic distortion from LNA circuit

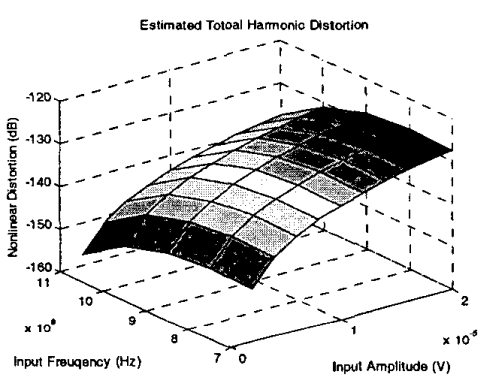

Fig. 9. Estimated total harmonic distortion from macromodel

Fig. 8 and Fig. 9 depict the total harmonic distortion from the original LNA circuit and that from the macromodel respectively as a function of the input amplitude and the input frequency. The "exact" values in Fig. 8 represent the exact distortions calculated by Harmonic Balance method. The maximum absolute error between Fig. 8 and Fig. 9 is less than $0.02 \mathrm{~dB}$, which demonstrates the accuracy of the LNA model under single-tone inputs over a reasonably wide frequency range.

In order to test the simulation speed of the macromodel, we run steady-state simulation on both the LNA circuit and its macromodel. The computation costs are 1.71 seconds for the original LNA circuit and 0.0047 seconds for the macromodel respectively.

\section{B. Double-Balanced Mixer}

Fig. 10 shows the double-balanced mixer in the GSM receiver system, which is used in this subsection to verify the macromodel structure in Fig. 5. We consider the down-conversion case. The amplitude and frequency ranges for the RF input are $10 \mu \mathrm{V} \sim 50 \mu \mathrm{V}$ and $935 \mathrm{MHz} \sim 960 \mathrm{MHz}$ respectively. For the LO input, its amplitude and frequency are varied from $0.8 \mathrm{~V} \sim 1 \mathrm{~V}$ and $864 \mathrm{MHz}-889 \mathrm{MHz}$ respectively. The maximum value of $i$ and $j$ in Fig. 5 is set to 3 , and the transfer function order in the macromodel is set to 5 . There are 19 signal paths included in the mixer model. We run steady-state analysis to test the macromodeling accuracy. The maximum, minimum and average modeling error are respectively $6.92 \%, 2.47 \%$ and $4.62 \%$.

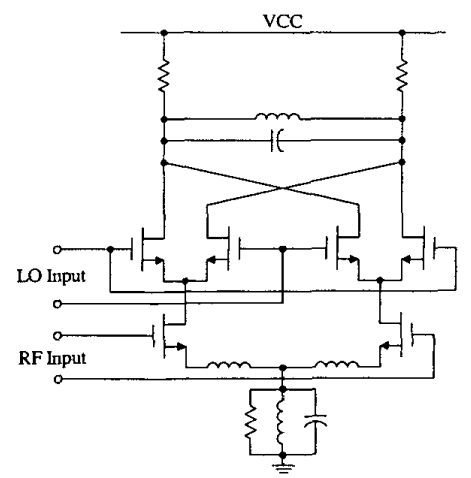

Fig. 10. Circuit schematic of mixer

In order to investigate the strongly nonlinear behavior of mixer circuits, we rebuild the mixer model with different options. TABLE I shows the average modeling errors where the signal paths containing signum functions $\operatorname{sign}(L O)$ and $\operatorname{sign}(R F)$ are included or excluded. Two observations can be made from the data in TABLE I. First, the average modeling error more than doubles if the signum function $\operatorname{sign}(L O)$ is excluded. It, in turn, indicates that the signum function $\operatorname{sign}(L O)$ in Fig. 5 plays a significant role in the modeling accuracy, and the mixer circuit presents strong nonlinearities in terms of its LO input. Secondly, the average modeling error is almost unchanged if the signum function $\operatorname{sign}(R F)$ is also used. This implies that the mixer circuit only exhibits weakly nonlinear behaviors for its RF input and it is not necessary to include strongly nonlinear functions for RF input in our macromodel.

TABLE I. MOdeling Result For THE Mixer CIRCUIT

\begin{tabular}{ccc}
\hline $\operatorname{sign}(L O)$ & $\operatorname{sign}(R F)$ & Average Error \\
\hline Include & Exclude & $4.62 \%$ \\
\hline Exclude & Exclude & $9.44 \%$ \\
\hline Include & Include & $4.42 \%$ \\
\hline
\end{tabular}

The macromodel described above can be further simplified since a signal path is negligible if its relative gain is smaller than a user-specified threshold $\varepsilon$. The relative gain of ith signal path is defined as

$$
G_{i}^{R}=\frac{G_{i}}{M A X\left(G_{i}\right)}
$$

where $G_{i}$ is the absolute gain of ith path and $\operatorname{MAX}\left(G_{i}\right)$ is the maximum absolute gain of all signal paths. For $\varepsilon=1 \%, 13$ signal paths are rejected and we obtain a new mixer model only containing 6 paths. All nonlinear signal paths corresponding to even order distortions are neglected in this example because the even order distortions should be very small for the double-balanced mixer in Fig. 10. The average error of such a simplified macromodel is $4.64 \%$, which is almost identical to that of the original 19-signalpath model.

For testing the simulation speed of the macromodel, we run steady-state simulation on both the mixer circuit and its macromodel. The computation costs are 10954 seconds for the original mixer circuit and 0.0247 seconds for the macromodel respectively.

C. IF Amplifier

The IF amplifier in the GSM receiver system is a bandpass 
filter, whose small-signal gain is $25 \mathrm{~dB}$ at the center frequency $71 \mathrm{MHz}$. In this paper, we only model the ideal functionality of the IF amplifier by a linear transfer function, since our interest focuses on the nonlinearities introduced by the low noise amplifier and the RF mixer. In system-level simulations, it is necessary to use different level models for different component blocks. In such a way, many important tradeoffs can be explored at early design stages.

D. System-level simulation

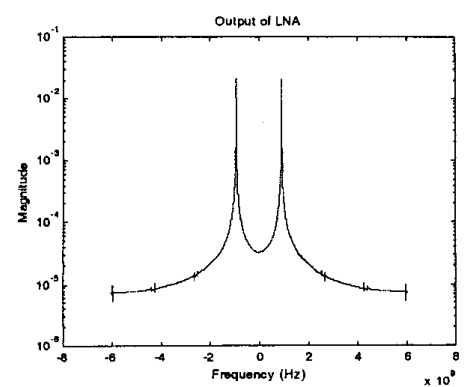

Fig. 11. Output frequency spectra of low noise amplifier

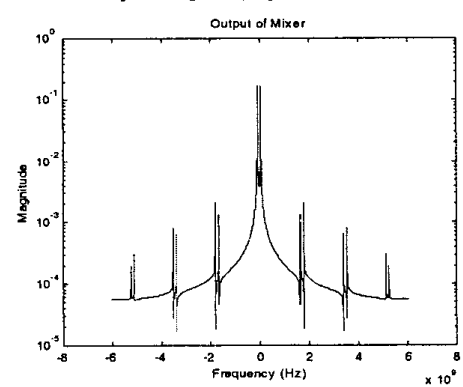

Fig. 12. Output frequency spectra of mixer

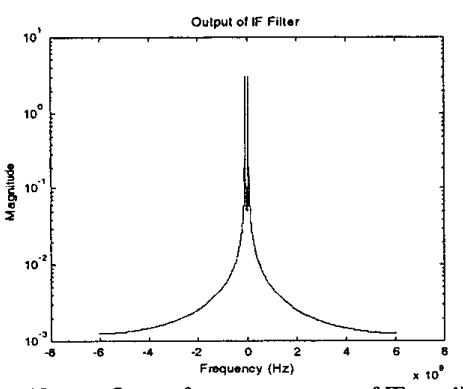

Fig. 13. Output frequency spectra of $\mathrm{IF} \times 10^{\circ}$ amplifier

We connect the macromodels for all these components together and construct a signal flow graph in our system-level simulation tool. A transient simulation is run for such a signal-flowgraph-based GSM receiver in time domain $[0,1 \mu s]$. Then, we perform FFT on the output signal of the low noise amplifier after the steady state is reached. Fig. 11 gives the frequency spectra of the LNA output, whose fundamental frequency is $935 \mathrm{MHz}$. In Fig. 11 , the higher order harmonic distortion is almost invisible. This observation is consistent with the results in Fig. 8 and Fig. 9, where the total harmonic distortion is less than $-120 \mathrm{~dB}$ in the current case. After the LNA output passes through the mixer, the RF signal is transferred to IF signal in Fig. 12, whose fundamental frequency is $71 \mathrm{MHz}$. Note that a great number of higher order harmonics are generated by the mixer, because the mixer circuit is a strongly nonlinear system due to the large LO input. Finally, those higher order harmonics from the mixer are filtered out by the IF amplifier and we obtain a clear IF signal as shown in Fig. 13.

For the transient simulation in $[0,1 \mu s]$, the overall computation time is 36.21 seconds. Note that, by employing the macromodels, the system-level simulation is quite efficient and can enable efficient system-level exploration and verification.

\section{Conclusions}

Modeling and representing nonlinear behaviors of RF circuits by simple macromodels is challenging due to the numerous nonlinear components that contribute to the overall circuit performance. In this paper, a frequency-separation approach is proposed to generate simple high-level macromodels ( 3 signal paths for LNA and 6 signal paths for mixer) for both weakly nonlinear and strongly nonlinear RF circuits. By employing these macromodels, system-level simulations can be accomplished with rarely low computation cost, as demonstrated by our GSM receiver system example. Using frequency separation we are able to generate an efficient extension of the more conventional macromodels based on Volterra kernel calculations.

\section{Acknowledgements}

This work has been supported by the MARCO Center for Circuits, Systems and Software (under MARCO contract 2001-CT888 and DARPA grant MDA972-02-1-0004) and the Semiconductor Research Corporation (under contract 2000-TJ-779).

\section{Reference}

[1] P. Wambacq, G. Gielen and W. Sansen, "Interactive symbolic distortion analysis of analogue integrated circuits," IEEE European Conference on Design Automation, pp. 484-488, 1991.

[2] P. Wambacq and W. Sansen, Distortion Analysis of Analog Integrated Circuits, Kluwer Academic Publishers, Boston, 1998.

[3] P. Wambaca, G. Gielen, P. Kinget and W. Sansen, "High-frequency distortion analysis of analog integrated circuits," IEEE Trans. Circuits and Systems - II, vol. 46, pp. 335-345, Mar. 1999.

[4] P. Wambacq, P. Dobrovolny, S. Donnay, M. Engels and I. Bolsens, "Compact modeling of nonlinear distortion in analog communication circuits," IEEE Design, Automation and Test in Europe Conference and Exhibition, pp. 350-354, 2000.

[5] P. Dobrovolny, P. Wambacq, G. Bandersteen, S. Donnay, M. Engels and I. Bolsens, "Generation of multicarrier complex lowpass models of RF ICs," IEEE International Microwave Symposium Digest, pp. 413$422,2001$.

[6] M. Schetzen, The Volterra and Wiener Theories of Nonlinear Systems, J. Wiley \& Sons, 1980.

[7] D. Weiner and J. Spina, Sinusoidal Analysis and Modeling of Weakly Nonlinear Circuits, Van Nostrand Reinhold, 1980.

[8] I. Vassiliou and A. Sangiovanni-Vincentelli, "A frequency-domain, Volterra series-based behavioral simulation tool for RF systems," IEEE Custom Integrated Circuits Conference, pp. 21-24, 1999.

[9] T. Lee, The Design of CMOS Radio-Frequency Integrated Circuits, Cambridge University Press, 1998.

[10] B. Razavi, RF Microelectronics, Prentice Hall Inc., 1998.

[11] L. T. Pillage and R. A. Rohrer, "Asymptotic waveform evaluation for timing analysis," IEEE Trans. Computer-Aided Design, vol. 9, pp. 352-366, Apr. 1990.

[12] Peter Feldmann and Roland W. Freund, "Efficient linear analysis by Pade approximation via Lanczos process," IEEE Trans. ComputerAided Design, vol. 14, pp. 639-649, May. 1995.

[13] A. Odabasioglu, M. Celik and L. Pileggi, "PRIMA: passive reducedorder interconnect macromodeling algorithm," IEEE Trans. ComputerAided Design, vol. 17, pp. 645-654, Aug. 1998.

[14] H. Unbehauen and G. Rao, Identification of Continuous Systems, Elsevier Science Publishing Inc., 1987.

[15] X. Li, P. Li, Y. Xu, R. Dimaggio and L. Pileggi, "A frequency separation macromodel for system-level simulation of RF circuits", Technical Report, No. CSSI 02-39, Carnegie Mellon Univ., Oct. 2002. 